\title{
Renal salt-wasting syndrome associated with docetaxel in an esophageal cancer patient
}

\author{
Motoo Nomura $\cdot$ Minoru Kamata $\cdot$ Hiroyuki Kojima • \\ Satoshi Sawada
}

Received: 20 October 2011/Accepted: 16 December 2011/Published online: 25 January 2012

(C) The Japan Society of Clinical Oncology 2012

\begin{abstract}
Hyponatremia associated with syndrome of inappropriate secretion of antidiuretic hormone (SIADH) or renal salt-wasting syndrome (RSWS) after platinum-based chemotherapy is not uncommon. However, no reports of RSWS following taxane monotherapy have been previously published. We report a case of a 63-year-old man with esophageal cancer who developed RSWS after docetaxel administration. He was diagnosed as having esophageal cancer and underwent esophagectomy. This was followed by adjuvant chemoradiotherapy consisting of a total dose of 50 Gy plus cisplatin combined with 5-fluorouracil. Two years after primary treatment, he was admitted to our hospital because of severe pain due to bone metastasis and mediastinal lymph node metastases. Ten days following chemotherapy consisting of nedaplatin and 5-fluorouracil, he experienced severe hyponatremia $(113 \mathrm{mEq} / \mathrm{L})$ due to SIADH. Subsequently, 7 days following third-line chemotherapy with docetaxel, he again experienced severe hyponatremia $(104 \mathrm{mEq} / \mathrm{L})$, this time due to RSWS. He was treated with a sodium supplement, to which he responded well. He recovered from the hyponatremia episode within a few days without any complications. In summary, treatment with docetaxel may lead to RSWS, even in patients with normal renal function. To the best of our knowledge, this is the first reported case of RSWS related to taxane chemotherapy in esophageal cancer. Oncologists should be aware of the possibility that docetaxel could cause RSWS.
\end{abstract}

Keywords Renal salt-wasting syndrome - Docetaxel · Hyponatremia

M. Nomura ( $₫) \cdot$ M. Kamata $\cdot$ H. Kojima $\cdot$ S. Sawada Department of Radiology, Kansai Medical University, 2-3-1 Shinmachi, Hirakata, Osaka 573-1191, Japan e-mail: excell@hkg.odn.ne.jp

\section{Introduction}

Hyponatremia is among the metabolic disturbances oncologists confront in treating patients with malignant disease. Chemotherapy can precipitate or aggravate hyponatremia $[1,2]$. In several studies, hyponatremia associated with syndrome of inappropriate secretion of antidiuretic hormone (SIADH) or renal salt-wasting syndrome (RSWS) after platinum-based chemotherapy has been reported [3-6]. SIADH is characterized by hyponatremia caused by excessive secretion of ADH which causes excessive water reabsorption in the collecting ducts and a resulting dilutional hyponatremia. On the other hand, RSWS is caused by chemotherapy-related renal tubule damage which causes excessive excretion of sodium from the kidney. To the best of our knowledge, no reports of RSWS associated with docetaxel have been published. Herein we report the case of a 63-year-old man with esophageal cancer who developed RSWS after docetaxel administration.

\section{Case report}

A 63-year-old man was admitted to another hospital following a diagnosis of esophageal cancer by esophagogastroduodenoscopy and biopsy of an upper thoracic esophageal lesion in June 2008. As primary treatment, the patient underwent a subtotal esophagectomy with gastric tube reconstruction through the retrosternal route and a 3 -field lymphadenectomy. The proximal resection margin was positive, while the other resection margins were free of cancer cells. The stage was determined to be pT1bN0M0 according to the 6th edition of the American Joint Committee on Cancer tumor-node-metastasis staging system. He was discharged from that other hospital, and received 
sequential radiotherapy and chemotherapy. The radiation dose was $50 \mathrm{~Gy}$ in 25 fractions, followed by two cycles of chemotherapy consisting of cisplatin $80 \mathrm{mg} / \mathrm{m}^{2}$ on day 1 and continuous infusion of 5 -fluorouracil $800 \mathrm{mg} / \mathrm{m}^{2} /$ day on days $1-5$.

In January 2011, the patient was admitted to our hospital after initial consultation because of severe pain due to bone metastasis and mediastinal lymph node metastases. He received loxoprofen sodium dehydrate, tramadol, and lansoprazole orally. As secondary treatment, he received chemoradiotherapy (40 Gy in 20 fractions) consisting of a continuous infusion of 5 -fluorouracil $700 \mathrm{mg} / \mathrm{m}^{2} /$ day on days 1-5 and nedaplatin $120 \mathrm{mg} / \mathrm{m}^{2} /$ day on day 6 . Before chemotherapy, his estimated glomerular filtration rate was $82 \mathrm{~mL} / \mathrm{min} / 1.73 \mathrm{~m}^{2}$. Adequate hydration was ensured by administration of 2-3 L fluid per day for 3 days prior to nedaplatin administration to prevent nephrotoxicity. Ten days after initiation of chemotherapy, the patient developed severe malaise followed by a gradual disturbance of consciousness. He had severe hyponatremia $(113 \mathrm{mEq} / \mathrm{L})$, decreased serum osmolality ( $248 \mathrm{mOsm} / \mathrm{L})$, elevated osmolality $(688 \mathrm{mOsm} / \mathrm{L})$, an elevated urinary sodium concentration $(232 \mathrm{mEq} / \mathrm{L})$, and an elevated serum $\mathrm{ADH}$ concentration $(15.7 \mathrm{pg} / \mathrm{mL} ; 0.3-4.2 \mathrm{pg} / \mathrm{mL})$. Thyroid and adrenal functions were normal, there was no edema or body weight loss, and skin turgor was normal. Grade 1 nausea, which was assessed with National Cancer Institute-Common Terminology Criteria for Adverse Events (NCI-CTCAE) version 3.0, but no vomiting occurred after chemotherapy. Sodium intake was $245 \mathrm{mEq} /$ day and the amount of sodium excreted was $232 \mathrm{mEq} / \mathrm{day}$. These data strongly suggested SIADH. Severe hyponatremia was treated by infusion of $3 \%$ saline and fluid restriction. Serum sodium increased from 113 to $130 \mathrm{mEq} / \mathrm{L}$ during the first $48 \mathrm{~h}$, after which the patient's symptoms were improved.

Third-line chemotherapy with docetaxel $70 \mathrm{mg} / \mathrm{m}^{2}$ was initiated in April 2011. Before chemotherapy, his estimated glomerular filtration rate was $87 \mathrm{~mL} / \mathrm{min} / 1.73 \mathrm{~m}^{2}$. The patient complained of drowsiness and severe malaise 7 days after starting docetaxel administration. He had severe hyponatremia $(104 \mathrm{mEq} / \mathrm{L})$, decreased serum osmolality $(230 \mathrm{mOsm} / \mathrm{L})$, elevated osmolality $(519 \mathrm{mOsm} / \mathrm{L})$, and an elevated urinary sodium concentration $(45 \mathrm{mEq} / \mathrm{L})$. Thyroid, adrenal, and anterior pituitary functions were normal, and there was no edema or ascites. Grade 1 nausea but no vomiting occurred after chemotherapy. A CT scan of the brain showed no sign of metastases or cerebral edema. However, his urine volume was high $(>4500 \mathrm{~mL} /$ day $)$, and he experienced body weight loss and decreased skin turgor. The patient also exhibited evidence of volume depletion. Sodium intake was $120 \mathrm{mEq} /$ day and the amount of sodium excreted was $208 \mathrm{mEq} /$ day. Thus, he was diagnosed as having RSWS on the basis of dehydration and high urinary sodium excretion at onset. He recovered (serum sodium $134 \mathrm{mEq} / \mathrm{L}$ ) 4 days after developing the hyponatremic state, following treatment with normotonic saline and no fluid restriction. After his serum sodium level increased, his symptoms were improved. The patient refused to receive additional chemotherapy.

\section{Discussion}

Hyponatremia has been reported following the administration of anticancer drugs such as vincristine, vinblastine, cyclophosphamide, cisplatin, and carboplatin [1, 2]. Only one report is available on hyponatremia due to SIADH after administration of docetaxel for breast cancer [7]. However, no reports of RSWS following docetaxel monotherapy have been published. The present patient developed a sodium-wasting nephropathy after administration of docetaxel for esophageal cancer. Hypovolemic hyponatremia associated with RSWS is also seen in adrenal insufficiency. However, serum cortisol levels were appropriate for his state of illness. Furthermore, there was also no adrenal metastasis in our patient. The extrarenal salt loss (e.g., emesis or diarrhea) may cause hypovolemic hyponatremia, but gastrointestinal loss was not found. The most common causes of hyponatremia are congestive cardiac failure, cirrhosis, and nephrotic syndrome. These cases are associated with fluid overload and edema, but our patient had no features suggestive of those illnesses.

Both RSWS and SIADH share similar laboratory values, such as hyponatremia and increased urine sodium; however, the major diagnostic feature used to differentiate between these two syndromes is the excessive urinary excretion of sodium in RSWS, whereas patients with SIADH do not exhibit excessive excretion of sodium in the urine [2]. A diagnosis of RSWS was made when the amount of sodium intake and the amount of sodium excreted from the kidney were calculated. We summarize the major differential features for RSWS and SIADH in Table 1.

Table 1 Differential features for RSWS and SIADH

\begin{tabular}{lll}
\hline & RSWS & SIADH \\
\hline Findings & & \\
$\quad$ Hyponatremia & + & + \\
Dehydration & + & - \\
Urinary sodium excretion & ++ & - \\
$>$ sodium intake & & + \\
$\quad$ Increased secretion of ADH & \pm & Tumor, drug \\
Cause & Drug, nephritis & \\
Treatment & & + \\
$\quad$ Fluid restriction & - & - \\
Sodium supplement & + & \\
\hline
\end{tabular}


Chemotherapy-related renal proximal tubule damage is the most common cause of RSWS in patients receiving platinum-based chemotherapy [2-6]. The renal proximal tubules are the major site of sodium and water reabsorption. In the case of carboplatin, less renal toxicity was reported in comparison with cisplatin-induced RSWS [8].

Docetaxel is metabolized by the cytochrome P450 system and is excreted by bile into the feces, and renal excretion of unchanged docetaxel is minimal [9]. Nephrotoxicity associated with docetaxel is rare $(<0.1 \%)$. However, tubular nephrotoxicity associated with docetaxel in patients with normal renal function has been reported [10]. Those authors showed that the urinary $\beta 2$-microglobulin and $N$-acetyl- $\beta$-D-glucosaminidase were increased after docetaxel administration as well as cisplatin. Thus, RSWS can occur after administration of docetaxel, which induced renal tubule damage. Our patient had been previously treated with strongly nephrotoxic agents, such as cisplatin and nedaplatin. These previously administered platinum agents and a previous history of SIADH could contribute to this renal tubule damage induced by docetaxel, but this is unclear.

In summary, treatment with docetaxel may lead to RSWS, even in patients with normal renal function. To the best of our knowledge, this is the first reported case of RSWS related to taxane chemotherapy in esophageal cancer. Oncologists should be aware of the possibility that docetaxel could cause RSWS.

Conflict of interest The authors declare that they have no conflict of interest, financial interests, or funding considerations.

\section{References}

1. Raftopoulos H (2007) Diagnosis and management of hyponatremia in cancer patients. Support Care Cancer 15:1341-1347

2. Hamdi T, Latta S, Jallad B et al (2010) Cisplatin-induced renal salt wasting syndrome. South Med J 103:793-799

3. Kuhlmann MK, Burkhardt G, Köhler H (1997) Insights into potential cellular mechanisms of cisplatin nephrotoxicity and their clinical application. Nephrol Dial Transplant 12:2478-2480

4. Arany I, Safirstein RL (2003) Cisplatin nephrotoxicity. Semin Nephrol 23:460-464

5. Kröning R, Lichtenstein AK, Nagami GT (2000) Sulfur-containing amino acids decrease cisplatin cytotoxicity and uptake in renal tubule epithelial cell lines. Cancer Chemother Pharmacol 45:43-49

6. Daugaard G, Abildgaard U (1989) Cisplatin nephrotoxicity: a review. Cancer Chemother Pharmacol 25:1-9

7. Langer-Nitsche C, Lück HJ, Heilmann M (2000) Severe syndrome of inappropriate antidiuretic hormone secretion with docetaxel treatment in metastatic breast cancer. Acta Oncol 39:1001

8. Tscherning C, Rubie H, Chancholle A et al (1994) Recurrent renal salt wasting in a child treated with carboplatin and etoposide. Cancer 73:1761-1763

9. Shou M, Martinet M, Korzekwa KR et al (1998) Role of human cytochrome P450 3A4 and 3A5 in the metabolism of taxotere and its derivatives: enzyme specificity, interindividual distribution and metabolic contribution in human liver. Pharmacogenetics 8:391-401

10. Takimoto T, Nakabori T, Osa A et al (2011) Tubular nephrotoxicity induced by docetaxel in non-small-cell lung cancer patients. Int J Clin Oncol. doi:10.1007/s10147-011-0304-5 